\title{
A luminaire with variable light distribution
}

\author{
Marcin Leśko ${ }^{1, *}$, Antoni Różowicz ${ }^{2}$, Krzysztof Baran ${ }^{1}$, and Henryk Wachta ${ }^{1}$ \\ ${ }^{1}$ Rzeszow University of Technology, Faculty of Electrical and Computer Engineering, \\ al. Powstańców Warszawy 12, 35-959 Rzeszów, Poland \\ ${ }^{2}$ Kielce University of Technology, Faculty of Electrical Engineering, Automatic Control and \\ Computer Science, al. Tysiąclecia Państwa Polskiego 7, 25-314 Kielce, Poland
}

\begin{abstract}
Industrial facilities such as production and storage halls are a specific environment, which is usually characterized by a large usable area, which is managed in a way that brings together in the given area many functional zones for various purposes. Frequent changes in the management of industrial halls gives a need to change the lighting method. In such a situation, a lighting solution that enables the photometric characteristics of the luminaires to be adapted to variable development of the facility, covering not only the quantitative regulation of the luminous flux, but also the direction of its radiation into the environment, can be a helpful solution. The article presents an innovative concept of a luminaire, which makes it possible to change the light distribution without the need to replace optical elements. Simulation tests were carried out for the developed luminaire in order to check the possibility of obtaining the assumed distribution and shapes of light spots on the working plane. Thanks to the application of this solution in an environment where there is a need for frequent changes in the development of usable space requiring changes in lighting conditions, it is possible to dynamically adjust the characteristics of the installed system to new environmental conditions.
\end{abstract}

\section{Introduction}

Industrial facilities such as production and storage halls are a specific environment, which is usually characterized by a large usable area, which is managed in a way that brings together in the given area many functional zones for various purposes (fig. 1), like, for example, communication routes A, storage zones B or production zones $\mathrm{C}$. Due to the large dimensions of the building, lighting it in accordance with the guidelines of the standard requires the deployment of a large amount of lighting equipment with relatively high power, which is associated with significant investment and operating costs (energy consumption), which is not neutral for the environment. Due to the growing demand for electricity, and hence, the growing amount of pollution that gets into the environment as a result of energy production based on fossil fuels, there is a great pressure on the use of energy-saving technologies in all branches of the economy, also in lighting [4, 14]. The reduction of operating costs is influenced by many factors, such as: the degree of use of

* Corresponding author: mlesko@prz.edu.pl 


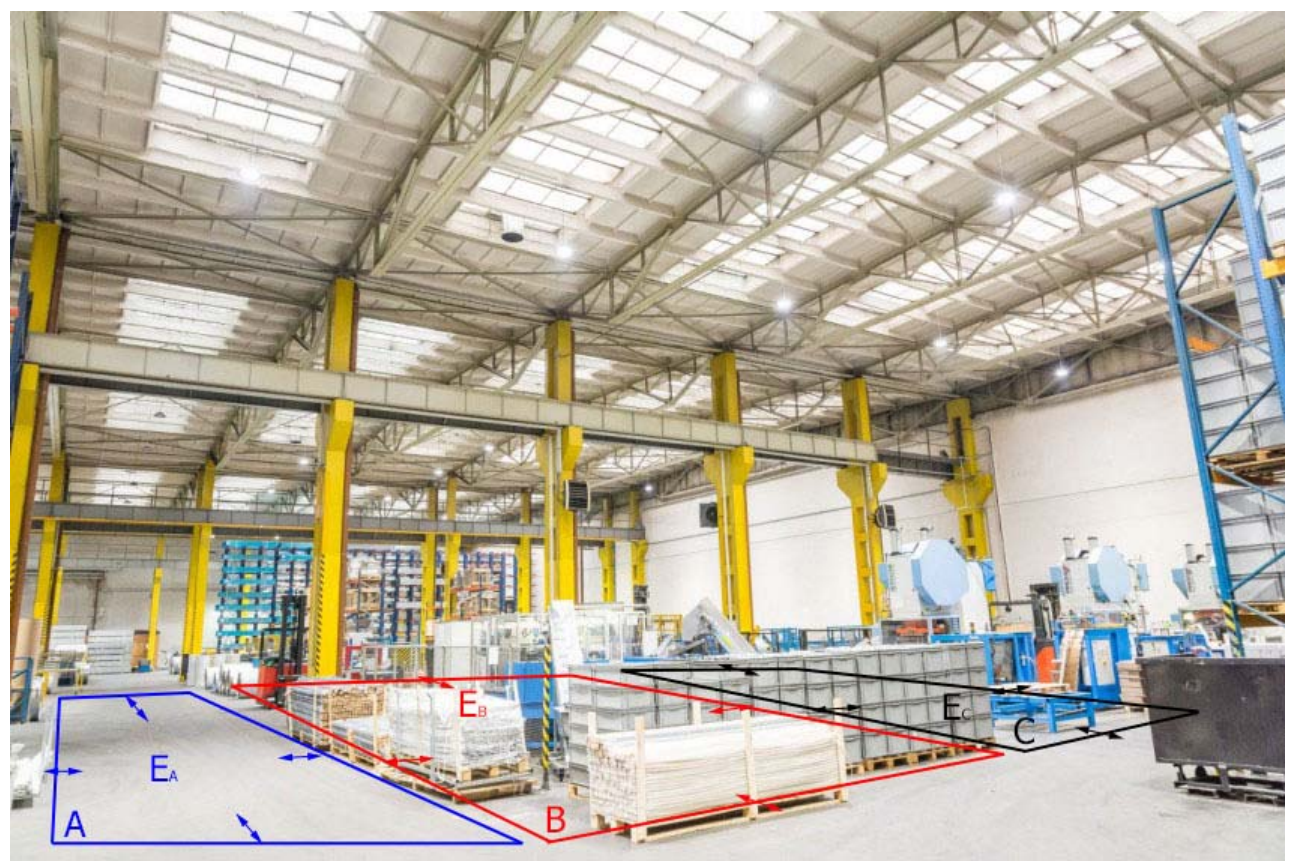

Fig. 1. View of an industrial hall with various designated functional zones.

daylight for lighting of halls, the use of luminaires with energy-saving sources and regulation of the luminous flux [3], and the use of adaptive lighting systems [2, 12, 13].

Currently, thanks to the use of semiconductor light sources technology, the implementation of this task is possible in a fairly wide range [6, 7]. LED sources also offer a relatively long lifetime, which is important when comparing the costs of source exchange, which causes temporary disorganization of the production cycle in the factory. These LED advantages are also used in other lighting areas, e.g. in floodlighting [9, 10, 18], where the costs of replacing hard to reach luminaires are similarly high.

This solution works very well in an environment in which a work zone with one function is set on a large area, which requires a certain level of illuminance. However, in the case where a lot of smaller zones with different functions are assigned in a given environment, which correspond to various required levels of illuminance, the situation becomes much more complicated. For example, in zones with a low required level of illuminance, there is an excess of light coming from a near neighboring zone with a high required level of illuminance. The second reason is the arrangement of luminaires of the same power throughout the hall. To some extent, this effect can be reduced by applying group control of the luminous flux of the luminaires, however, the functionality of the available lighting systems ends there. This is sufficient if the environmental conditions associated with the development of the hall are permanent. However, if the way of development changes during use, individual functional zones change their location, shape of the contour or sizes, lighting usually remains unchanged due to costs or logistic conditions. Also in the area of communication zones, it is possible to consider the potentiality of changing the lighting level. For low width routes, the need to achieve lower illuminance levels is usually not considered, however, when the specificity of production takes into account the transport of large-size elements, there is a need to provide significantly lower illuminance on surfaces for communication than on production areas. In addition, the communication routes can also be widened or narrowed and moved. 
In this situation, a special lighting system can be a good solution. It allows adjusting the photometric characteristics of luminaires to environmental conditions and variable development of the facility, comprising not only the quantitative regulation of luminous flux, but also the direction of its radiation. The purpose of this solution is not only to reduce energy consumption, but also to increase the effective use of energy for lighting purposes.

\section{The concept of a lighting system that implements a dynamically variable photometric characteristic}

In lighting of large spaces, such as industrial halls, highbay luminaires with rotational symmetrical luminous intensity distribution or two symmetry axes (linear luminaires) with different beam angles are usually used [15]. An innovative luminaire with dynamically variable light distribution characteristics maintains the functionality of existing solutions, and also provides the possibility of a smooth adjustment of the beam angle and enables an asymmetrical distribution. An important feature of this luminaire is to obtain these functions without the need to replace its optical system in order to obtain a specific type of distribution.

The innovative solution of the luminaire is based on the use of a set of light-optical elements involved in the production of light and forming the characteristics of its distribution by adjusting the operating parameters such as position, orientation, power, etc. The structure of the control and work system of the designed luminaire is shown in the figure 2 .

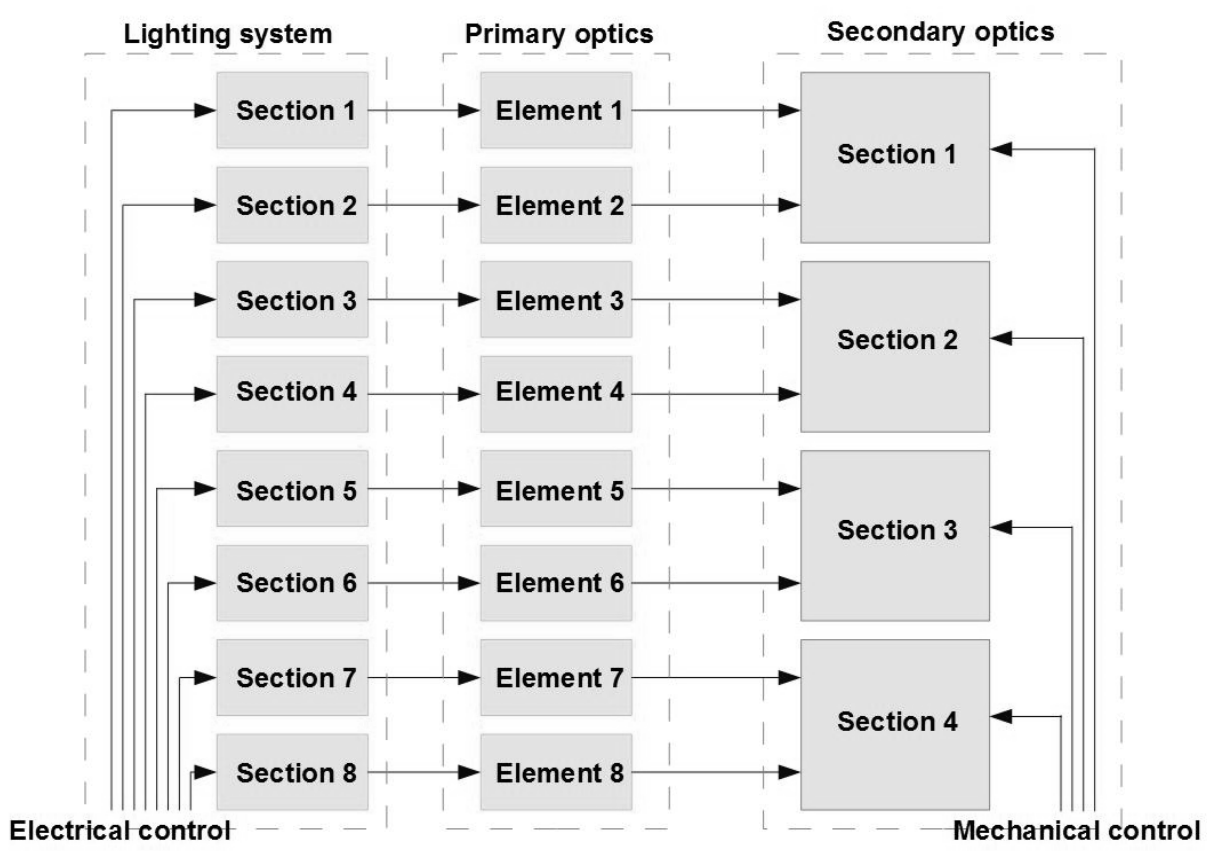

Fig. 2. Diagram of the lighting system's control structure.

In order to ensure the possibility of forming both symmetrical and asymmetrical distributions in any direction, regardless of the orientation of the luminaire, the whole lightoptical system has been divided into four main sections. Each section of the light-optical system consists of a matrix of LED sources, divided into two independently powered subsections, equipped with lenses as the primary optical system, whose task is to narrow the distribution of light sources and direct light towards the secondary optical system, 
which is a reflector. Matrix systems are often used lighting solutions in different lighting areas [19]. The secondary optical system is an element adapted to change the position by rotation about a horizontal axis passing through its base. This allows changing the direction of radiation from the luminaire, which changes the beam angle. The division of the lightoptical system into sections makes it possible to control the distribution of light symmetrically, asymmetrically and independently of each other, which allows extending or narrowing the beam angle only on one side, longitudinal, transverse or diagonal axis. The view of the luminaire is shown in the figure 3.

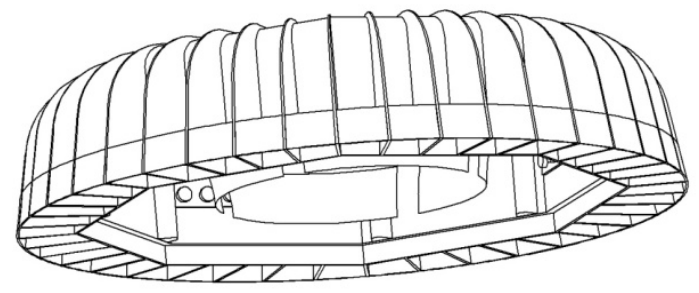

Fig. 3. View of the complete luminaire.

\section{Results of photometric analysis}

Forming luminous intensity distribution in currently used luminaires takes place through appropriately designed unadjustable optical systems that provide a specific type of distribution matched to work in a specific environment $[8,16]$. One type of luminaire can be used with various optical systems depending on the needs, and the change of light distribution takes place by exchanging elements of the optical system (lenses or reflectors), which are designed to form one specific type of light distribution $[11,17,20]$.

Ensuring the possibility of changing the light distribution by the optical system raises some construction problems, because usually the active elements, such as lenses or reflectors, have a shape optimized for a given type of distribution, thus different for wide distribution, and different for focused or asymmetrical [1]. Adjustment of the light distribution in the new luminaire is done by changing the position of the elements of the optical system with a fixed specific shape. One of the most important elements that determined the achievement of the assumed effects is the optical system. This issue came to find the right shape of the reflector, which will not only ensure the planned change of light distribution when changing its position, but also provide a proper uniformity of illuminance distribution on the working plane $[1,5]$.

Using the iterative methods in subsequent approximations, a reflector profile was developed. It generates a change in the beam angle within the assumed dimensions and range of angular motion between $80^{\circ}$ and $130^{\circ}$. The design of the reflector required correlating the shape of the focusing profile with the reflective properties of the material in terms of focusing and scattering of light, so that the reflector's uniform lighting distribution and the appropriate shape of the light spot can be obtained with different settings, and in addition for the reduction of luminous flux losses.

Computer simulations were carried out in order to check the possibility of obtaining the assumed types of distribution and shapes of light spots on the working plane. Analysis of lighting conditions was carried out for a horizontal calculation plane with dimensions of 10 by $10 \mathrm{~m}$ and a luminaire placed above it at a height of $4 \mathrm{~m}$. Exemplary results of light distribution curves and light spots on the working plane obtained for various settings of luminaire are presented below. 
Figure 4 shows the rotationally symmetrical wide beam and the corresponding light spot. The basic functionality of the luminaire enabling the change of the beam angle is ensured.

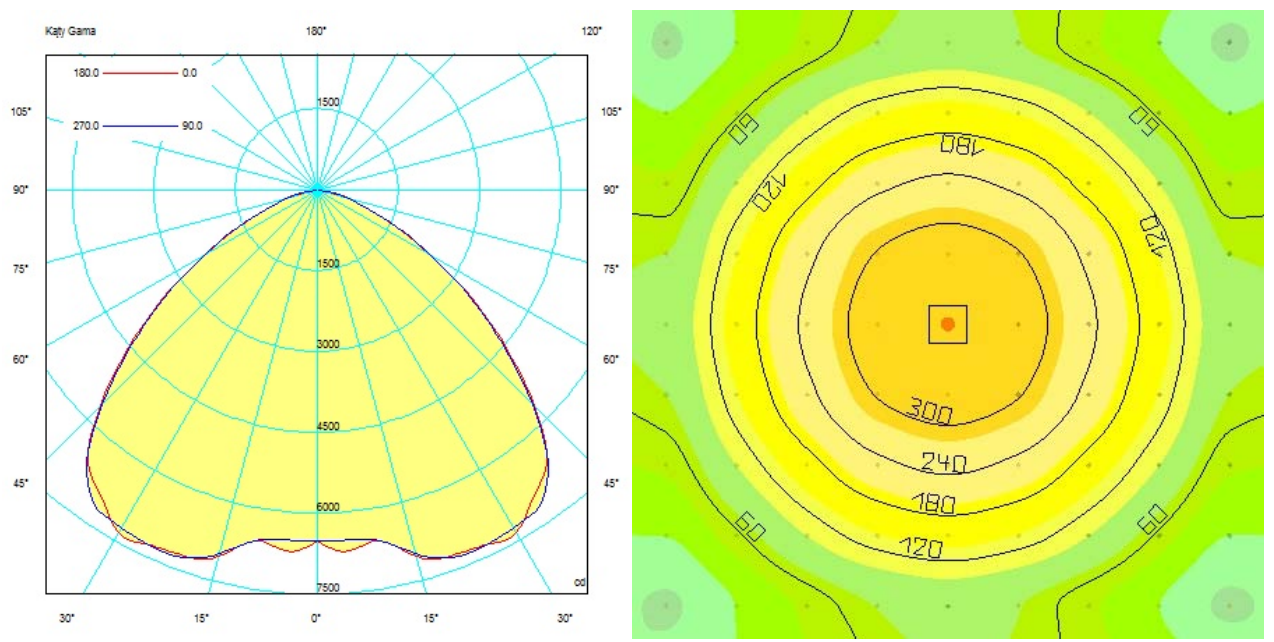

Fig. 4. Luminous intensity distribution curve and illuminance distribution on the plane for the wide symmetrical distribution option.

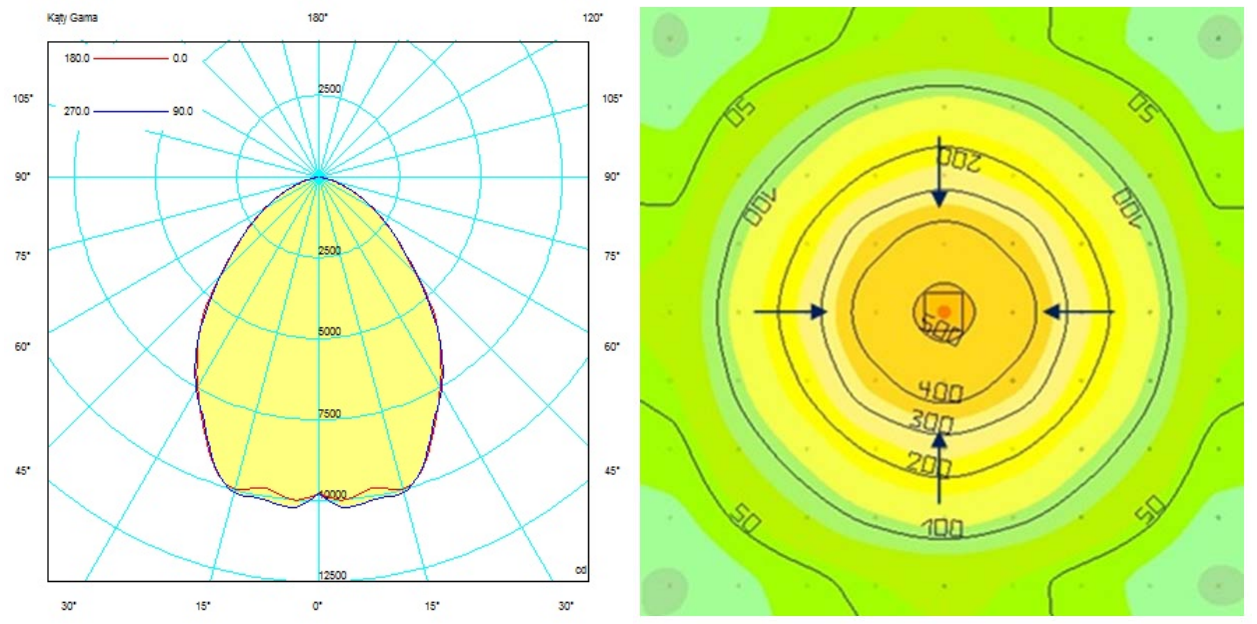

Fig. 5. Luminous intensity distribution curve and illuminance distribution on the plane for the narrow symmetrical distribution option.

Figure 5 shows a narrow beam with a light spot, where an increase of the illuminance levels in the center of the plane under the luminaire is obtained. Figure 6 presents the results for narrowing the basic distribution in one axis, which results in a distribution with two axes of symmetry, as in a linear luminaire. The light distribution was extended in the longitudinal axis and narrowed in the transversal axis. 

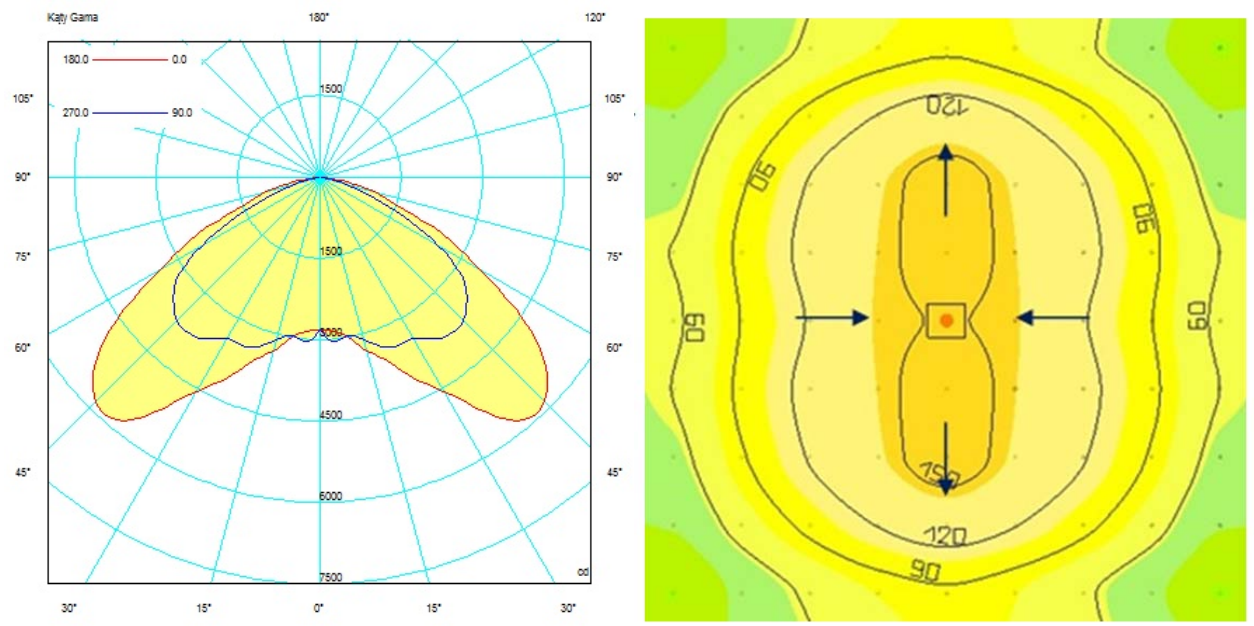

Fig. 6. Luminous intensity distribution curve and illuminance distribution on the plane for the bi-axial symmetrical distribution option.

Figure 7 shows the results for narrowing the light distribution one-sidedly against the basic distribution, whereas figure 8 also shows an asymmetrical distribution, which is more emphasized in one of the directions. This causes the light spot to be shifted on the plane in one axis. The maximum illuminance no longer lies in the saucer of the suspension height of the luminaire.

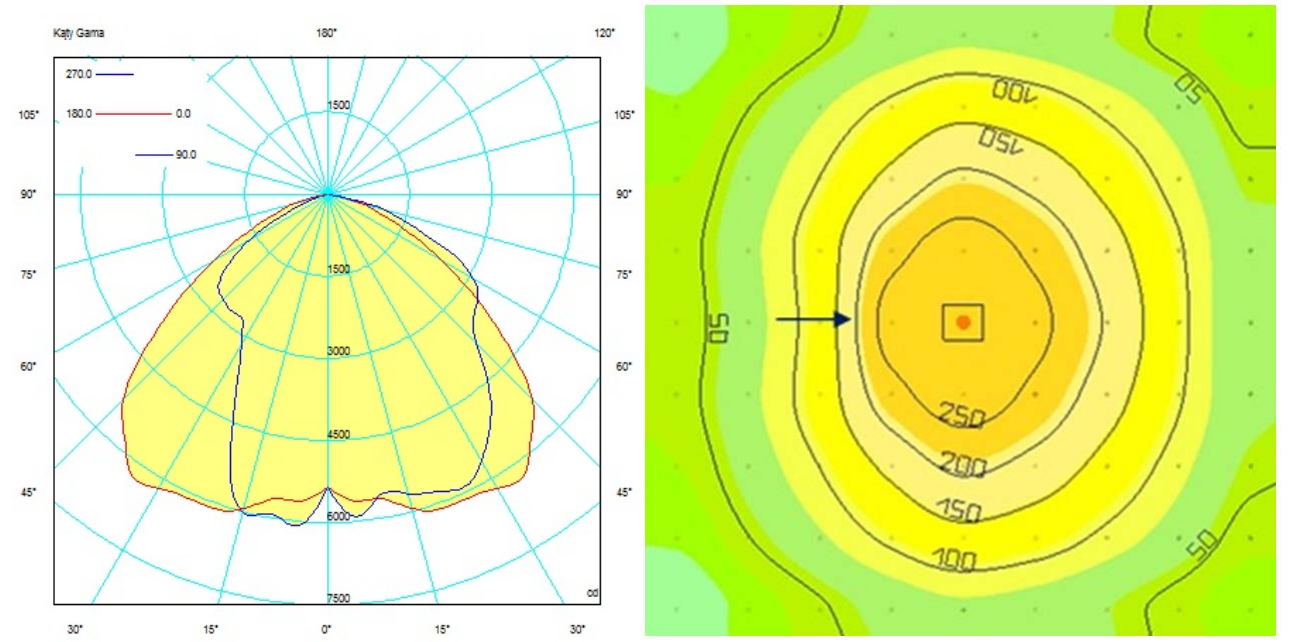

Fig. 7. Luminous intensity distribution curve and illuminance distribution on the plane for the asymmetrical unilaterally narrowed distribution option. 

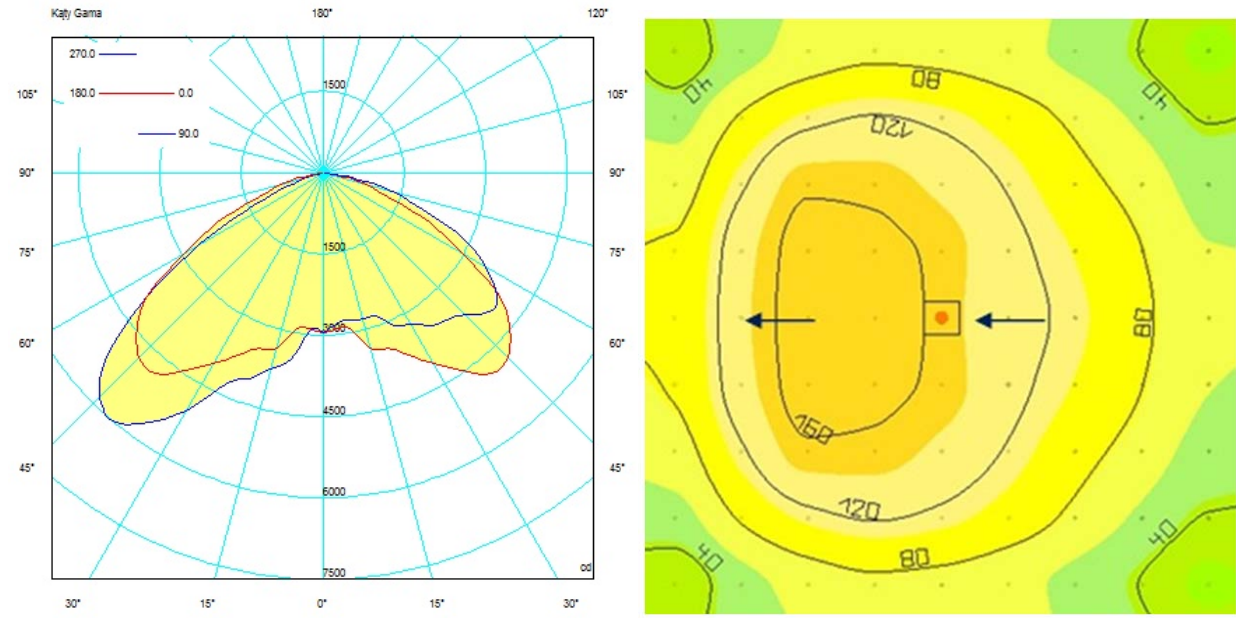

Fig. 8. Luminous intensity distribution curve and illuminance distribution on the plane for the asymmetrical distribution option, with a shift in the direction of maximum radiation in one axis.

Figure 9 shows the shift of the light spot in two axes, which translates into the displacement of the maximum illuminance in the corner of the illuminated working plane.

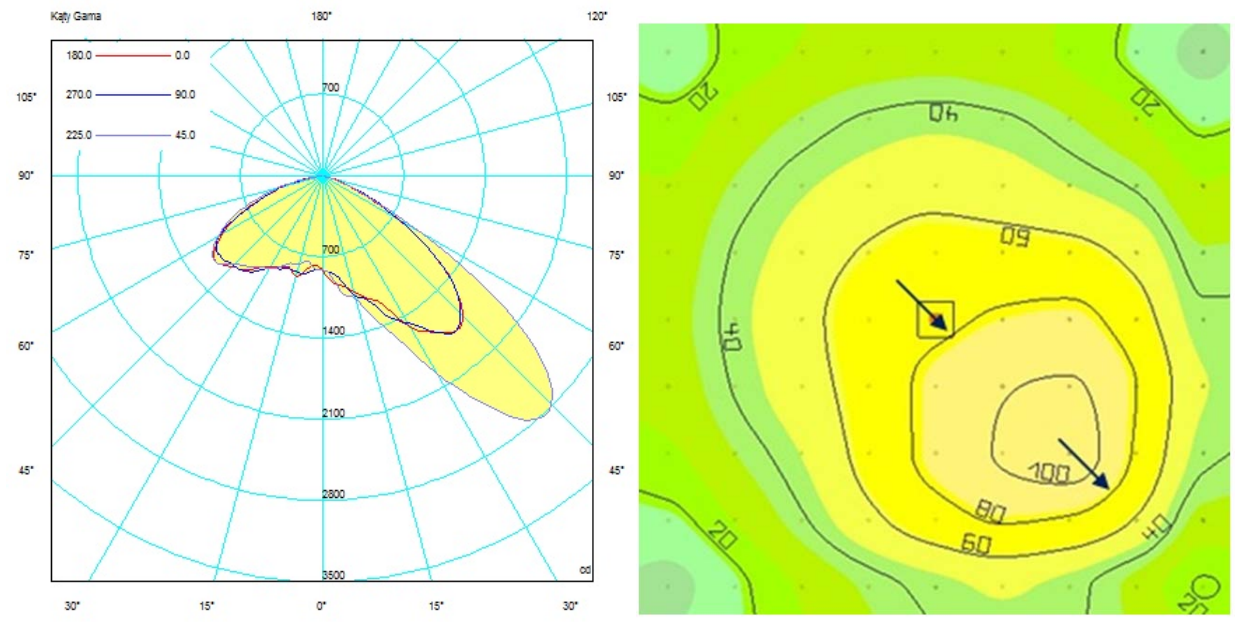

Fig. 9. Luminous intensity distribution curve and illuminance distribution on the plane for the asymmetrical distribution option, with a shift in the direction of maximum radiation in two axes.

\section{Conclusion}

The presented concept of a lighting system based on a luminaire with an innovative optical system enables the implementation of dynamically variable photometric characteristics of the luminaire. Thanks to this in an environment where there is a need for frequent changes in the development of the usable space requiring changes in lighting conditions, it is possible to dynamically adjust the characteristics of the installed system to new environmental conditions without the need to modernize it and interfere with the hardware structure. The necessary changes are introduced by the software using the control system and the user interface. Thanks to this, the time needed to adapt the lighting to the new 
requirements is significantly shortened and the costs of modernization are reduced because there is no need to replace the lighting equipment.

The developed lighting and optical system of the luminaire fulfills the constructional assumptions regarding the possibility of changes in light distribution characteristics consisting of its narrowing and extending and moving the direction of light radiation beyond the center of the luminaire mounting height, thus also realizing asymmetrical distribution in various configurations. This allows it to be used in a lighting system to dynamically create lighting conditions depending on the momentary needs, which ensures great flexibility in its operation.

The presented solution and the results obtained by simulation are the first stage of the research. The next step is to analyze the group work of luminaires. At the same time, work is carried out on hardware implementation of the designed solution. The luminaire with variable light distribution is one of the components of the adaptive lighting system, on which parallel research are carried out.

The research was carried out as part of the project titled „Development of the Intelligent Light Distribution System SOLLS ACTIVE SKY" by In4Poland Sp. z o.o., Intelligent Development Operational Program 2014-2020, suboperation 1.1.1. "Industrial research and development works carried out by enterprises", co-financed by the European Regional Development Fund. The presented solution is the subject of patent application No. P.421299.

\section{References}

1. C. Cuttle, Lighting Design: A Perception-Based Approach Routledge, (2015)

2. S.I. Hong, C.H. Lin, An efficient ILACS control algorithm for intelligent LED indoor lighting system 2017 19th International Conference on Advanced Communication Technology (ICACT), IEEE, (2017)

3. C. Huang, X. Zhang, Impact and feasibility of darklight LED on indoor visible light positioning system 2017 IEEE 17th International Conference on Ubiquitous Wireless Broadband (ICUWB), IEEE, (2017)

4. A. Jayawardena, D. Duffy, J.M. Manahan, Lighting Matters in Industrial Environments: A Framework Linking Workplace Safety to Lighting Quality Metrics IEEE Industry Applications Magazine, 23(3), 54-63, (2017)

5. M. Karlen, J.R. Benya, C. Spangler, Lighting Design Basics, (2nd Edition Wiley, 2012)

6. T. Khanh, P. Bodrogi, Q.T. Vinh, H. Winkler, Led Lighting. Technology and Perception (Wiley, Weinheim, 2015)

7. V.K. Khanna, Fundamentals of Solid-State Lighting: LEDs, OLEDs, and Their Applications in Illumination and Displays CRC Press, (2015)

8. R.J. Koshel, Illumination Engineering: Design with Nonimaging Optics Wiley-IEEE Press, (2013)

9. R. Krupiński, Dynamically Variable Luminance Distribution as the Method of Designing and Architectural Floodlighting Proceedings of 2016 IEEE Lighting Conference of the Visegrad Countries (Lumen V4), IEEE, (2016)

10. R. Krupiński, Visualization as alternative to tests on lighting under real conditions Light \& Engineering, 23, No. 4, 33-40, (2015).

11. S. Liu, X. Luo, Led Packaging for Lighting Applications. Design, (Manufacturing and Testing Wiley, Chemical Industry Press, 2011) 
12. S.A. Mahajan, S.D. Markande, Design of intelligent system for indoor lighting 2016 International Conference on Computing Communication Control and automation (ICCUBEA), IEEE, (2016)

13. P. Mandal, B. Roy, Adaptive dimming scheme based daylight pipe integrated indoor lighting system under Perez All-weather sky model 2016 IEEE International WIE Conference on Electrical and Computer Engineering (WIECON-ECE), IEEE, (2016)

14. L. Martirano, S. Di Ponio, Procedure to Evaluate Indoor Lighting Energy Performance 2012 11th International Conference on Environment and Electrical Engineering, IEEE, (2012)

15. M.S. Rea, The IESNA Lighting Handbook. Reference \& Application IESNA, New York, (2000)

16. A. Różowicz, M. Leśko, H. Wachta, Evaluation of the zonal luminous flux distribution of LED sources Proceedings of 2016 13th Selected Issues of Electrical Engineering and Electronics (WZEE), IEEE, (2016)

17. A. Różowicz, M. Leśko, H. Wachta, The technical possibilities of losses reduction in the LED optical systems Proceedings of 2016 IEEE Lighting Conference of the Visegrad Countries (Lumen V4), IEEE, (2016).

18. K. Skarżyński, Field measurements of Floodlighting Utilisation Factor Proceedings of 2016 IEEE Lighting Conference of Visegrad Countries (Lumen V4), IEEE, (2016)

19. S. Słomiński, Potential resource of mistakes existing while using the modern methods of measurements and calculation in the glare evaluation Proceedings of 2016 IEEE Lighting Conference of the Visegrad Countries (Lumen V4), (2016)

20. W.D. Van Driel, X.J. Fan, Solid State Lighting Reliability. Components to Systems Springer, (2013) 